\title{
Erratum
}

M. Burger · N. Monod

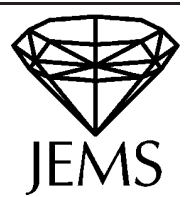

\section{Bounded cohomology of lattices in higher rank Lie groups}

\author{
J. Eur. Math. Soc. 1, 199-235 (1999)
}

An assumption is missing in the statement of our Theorems 1.1 and 1.2 in [1]. When considering a unitary representation $(\pi, \mathfrak{H})$ of a lattice $\Gamma$ in a product $G$ of Lie groups among which rank one factors occur, one has to suppose that the induced unitary representation $\operatorname{Ind}_{\Gamma}^{G}(\pi)$ of $G$ does not contain a subrepresentation which factors non-trivially through a rank one factor. Likewise, for Theorem 1.2, denoting by $G_{i}$ the closure of the canonical projection $\Gamma \rightarrow \operatorname{Aut} \mathcal{T}_{i}$, the induced unitary representation of $G_{1} \times G_{2}$ should not contain a subrepresentation factoring non-trivially through $G_{1}$ or $G_{2}$.

In the proofs given in [1], the omitted assumption was needed on pages 231 and 233 when claiming that $\widetilde{\mathfrak{H}}^{G_{i}}=0$.

In particular, Theorem 1.1 holds unchanged as soon as all factors have rank at least two. Notice also that the Theorems 1.1 and 1.2 hold without change for the trivial unitary representation on $\mathbf{C}$, and hence imply all corollaries stated in [1].

As stated originally, the Theorem 1.1 fails in the following interesting way: let $\Gamma$ be an irreducible cocompact lattice in $\mathrm{PSL}_{2}(\mathbf{R}) \times \mathrm{PSL}_{2}(\mathbf{R})$. Let $\left(\pi^{\prime}, \mathfrak{H}^{\prime}\right)$ be a non-trivial continuous unitary representation in the principal or complementary series of $\mathrm{PSL}_{2}(\mathbf{R})$. If we take $(\pi, \mathfrak{H})$ to be the unitary representation of $\Gamma$ defined by restricting $\left(\pi^{\prime}, \mathfrak{H}^{\prime}\right)$ to the projection of $\Gamma$ in the first factor, then we can prove (using yet unpublished calculations) that the kernel $E H_{\mathrm{b}}^{2}(\Gamma ; \mathfrak{H})$ of the natural map has dimension at least one.

Acknowledgement. This erratum arose from discussions with Yehuda Shalom, to whom we hereby express our appreciation.

\section{Reference}

1. M. Burger, N. Monod: Bounded cohomology of lattices in higher rank Lie groups. J. Eur. Math. Soc. 1(2), 199-235 (1999)

M. Burger, N. Monod: ETHZ, Department of Mathematics, Rämistrasse 101, CH-8092 Zürich, Switzerland, e-mail: \{burger,monod $\} @$ math.ethz.ch 